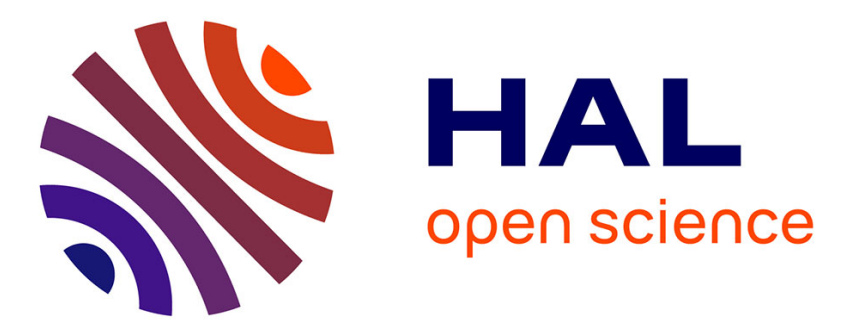

\title{
Confinement, entropic effects and hydrogen bond network fluctuations of water in Nafion membrane
}

Marie Plazanet, Renato Torre, Francesco Sacchetti

\section{To cite this version:}

Marie Plazanet, Renato Torre, Francesco Sacchetti. Confinement, entropic effects and hydrogen bond network fluctuations of water in Nafion membrane. Journal of Molecular Liquids, 2016, 219, pp.11611164. 10.1016/j.molliq.2016.01.079 . hal-01583242

\section{HAL Id: hal-01583242 \\ https://hal.science/hal-01583242}

Submitted on 7 Sep 2017

HAL is a multi-disciplinary open access archive for the deposit and dissemination of scientific research documents, whether they are published or not. The documents may come from teaching and research institutions in France or abroad, or from public or private research centers.
L'archive ouverte pluridisciplinaire HAL, est destinée au dépôt et à la diffusion de documents scientifiques de niveau recherche, publiés ou non, émanant des établissements d'enseignement et de recherche français ou étrangers, des laboratoires publics ou privés. 


\title{
Confinement, entropic effects and hydrogen bond network fluctuations of water in Nafion membrane
}

\author{
Marie Plazanet ${ }^{\mathrm{a}, \mathrm{b}, 1}$, Renato Torre ${ }^{\mathrm{c}}$, Francesco Sacchetti ${ }^{\mathrm{a}}$ \\ ${ }^{a}$ Dip. di Fisica, Università degli Studi di Perugia, I-06123 Perugia, Italy \\ ${ }^{b}$ CNRS and Univ. Grenoble Alpes, LIPhy, F-38000 Grenoble, France \\ ${ }^{c}$ European Laboratory for Non-Linear Spectroscopy (LENS) and Dip. di Fisica ed \\ Astronomia, Università di Firenze, I-50019 Sesto Fiorentino, Firenze, Italy
}

\begin{abstract}
Nafion ${ }^{\circledR}$ is, to date, the most widely used polymeric material as Proton Exchange Membrane. The complex structural organization of this amphiphilic polymer leads to unique physical properties, especially the proton conductivity, which is related to the properties of water confined in the hydrophilic nanochannels. At subzero temperatures, the water remains mobile down to $\sim 200 \mathrm{~K}$, desorbing and re-sorbing reversibly in and out of the membrane depending on the temperature. We therefore investigated the structure of water/ice by neutron diffraction and Compton spectroscopy in order to check if some particularity in the hydrogen bond network was at the origin of the sorption/desorption phenomenon. No anomaly of the ice Bragg diffraction pattern is detected as a function of the temperature, indicating that the melting of ice is entirely driven by the membrane. The Compton profiles of water confined in Nafion at room temperature, instead, exhibit tiny differences with respect to bulk water. These differences increase on lowering the membrane
\end{abstract}

\footnotetext{
*corresponding author: marie.plazanet@unipg.it, Tel. +39 0755852705
} 
hydration. The Compton investigations therefore show fluctuations in the hydrogen bond geometry of water that could either be due to confinement effect or to the presence of hydronium ions in the liquid.

Keywords: Nafion, confined liquid, Neutron Scattering Transient Grating Spectroscopy, Compton Spectroscopy

\section{Introduction}

The investigation of confined water attracts a wide research interest because of its ubiquitous presence in many systems of different nature. Porous materials such as templated silicates, polymeric membranes, polymers with intrinsic microporosity, are all more or less hydrophilic systems that can be filled with water and used for example for filtration, or may be exposed to water vapour if used for gas separation or catalysis [1]. The other example of paramount importance is the one of biological systems, that always contain water confined in an overcrowded media of protein, lipids or polysaccharides, like water in cell or hydration water of proteins [2]. Confined water shows complex structural and dynamic features that are strongly dependent on the nature and dimension of confinements [3-5]. Moreover, in most confining environments, water is not pure but contains ions released by the surrounding matrix, so the studied systems are confined electrolytes instead of confined water. This is certainly true in biological systems or soft condensed matter, in which (poly)electrolytes such as lipids, DNA etc. contain acid groups releasing protons or ions in the hydration water.

This is also the case of Nafion ${ }^{\circledR}$. This well known polymer is widely used in the form of protons exchange membranes for fuel cells $[6,7]$. Nafion is 
an amphiphilic polymer made of fluorinated chains carrying hydrophilic sulfonate $\left(\mathrm{SO}^{-}\right)$anions. The resulting structure is a complex polymer organization in which the hydrophilic side chains are organized to form a network of channels and cavities that water fills upon hydration. Several models have been proposed for the structural organization, in which more or less ordered nanometric (2-6 nm) cavities are interconnected by channels [8-13] enabling the water transport through the membrane, leading to a very good proton conduction [14]. The membrane swells upon hydration, protons are released in water by the sulfonate groups and the membrane results to be filled with an aqueous solution of hydronium ions, the concentration of which depends on hydration. This system, besides its high importance in industrial application, is a very interesting one for the study of nanofluidics of liquid and/or water electrolytes.

In our previous studies, we focused on the behaviour of water in Nafion membranes below the ice melting point $[15,16]$. Using Transient Grating spectroscopy, we could monitor the formation of ice below $0^{\circ} \mathrm{C}$, already pointed out by earlier studies [17-20]. The ice formation on the membrane surface is caused by water desorption out of the membrane itself, in a progressive way down to $\sim 200 \mathrm{~K}$. Our quantitative measurements prove the full reversibility of the process: as soon as the temperature rises, the ice melts and water is adsorbed back into the membrane.

Combining the Transient Grating data with neutron diffraction, we could also address several questions [16]. First, the ice formed during the cooling crystallizes in micrometric-size crystallites, as shown by the characterization of the acoustic wave propagation, and in the regular hexagonal $I_{h}$ phase, as 
proved by neutron diffraction investigations. Moreover, we proposed a simple physical model that is able to describe the ice formation process during cooling (or ice melting during heating) and the water desportion phenomena taking place in Nafion membranes (respectively sorption). The model is based on the freezing point depression caused by the presence solutes $\left(\mathrm{H}_{3} \mathrm{O}^{+}\right.$ ions) in water and the confinement in nanocavities. The quantitative agreement between model and data proves the entropic origin of the persistence of water mobility down to $\sim 200 \mathrm{~K}$.

Eventually, in comparing the freezing point depression of the solution to the one of confined water in Nafion membrane, according to Gibbs-Thomson equation for confined liquids [3], we could reconcile both effects on different timescales: upon cooling, within the Gibbs-Thomson limit, the water is maintained liquid thanks to the confinement. Then the system equilibrate the membrane hydration in order to equal the chemical potential of the solution inside with the one of bulk ice formed on the membrane surface. This equilibrium is obtained by water desorption out of the membrane and migration toward the surface, leading to ice formation upon cooling. Because of the reduced mobility of water below $0^{\circ} \mathrm{C}$, the kinetics of this phenomenon are slow, the equilibrium is typically reached on timescales of tens of minutes. If the system is quickly cooled down to a temperature which is below the Gibbs-Thomson limit, then the water freezes instantaneously inside the membrane, like is observed when highly hydrated membranes are quenched to 200K. [21]. The various scenarios are summarized in the figure 1: the regions limited by the confinement and solution melting point depression curves are, in semi-quantitive agreement with various studies [15, 16, 19-21]. 

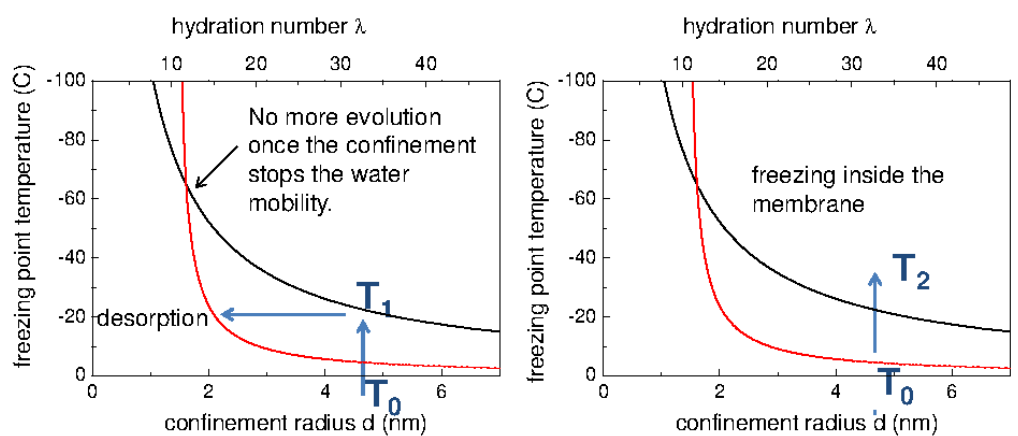

Figure 1: The two similar figures show the freezing point of the $\mathrm{H}_{3} \mathrm{O}^{+}$solution as a function of characteristic confinement radius. The black (solid) line is given by the GibbsThomson depression of the freezing point of a liquid due to confinement [21], while the red (dashed) line is the freezing point of an ideal solution of concentration varying with the confinement radius. The figure presents two scenarios. Left: the sample hydrated at $\lambda=30$ is cooled down from an initial temperature $T_{0}>0^{\circ} \mathrm{C}$ to $T_{1}=-20^{\circ} \mathrm{C}$ : the confined water is still above the freezing point, and desorbs to bring the concentration to the freezing limit of the solution. The sample desorbs until $\lambda=13$. Right: from $T_{0}$, the same sample to cooled down to $T_{2}=-40^{\circ} \mathrm{C}$, and the water freezes inside the membrane. No desorption is observed. 
These former studies tend to show that the electrolyte nature of the confined solution is responsible for the water mobility at sub-zero temperatures. However, as previously discussed [16], not all systems containing confined electrolyte exhibit the sorption/desorption phenomenon. The desorption is at first sight a combination of the confinement effect, the entropy related to the presence of charges and the elasticity of the confining environment. However, more subtle effects such as particularities of the hydrogen bond network may also be involved. Such a particularity, in the case of Nafion, may also be responsible for the exceptional proton conductivity which still remains unexplained. IN this context, we started to investigate the distortion of the hydrogen bond network and ground state of the protons by means of X-ray Compton Spectroscopy (CS). CS is a direct probe of the momentum distribution of valence electrons, therefore a quantitative measurement of electronic density in chemical bonds, particularly suitable for the investigation of the geometry of hydrogen bonds $[22,23]$. The observation of differences in Compton profiles (CP) measured in confined and bulk water is a direct probe of the fluctuations of the hydrogen bond network induced by confinement [24].

In the present paper, we address the question of the distortion of the tetrahedral structure of water in hydrated Nafion membrane. First, we reexamine the diffraction patterns measured by Neutron Scattering and their temperature dependence in order detect some specific behaviour of the $I_{h}$ lattice parameters related to the melting of ice upon heating above $200 \mathrm{~K}$. Then, we present our first results of X-ray Compton investigations on Nafion membranes at various hydration levels in order to characterization the liquid 
state. We discuss them in terms of variation with respect to bulk water due to to confinement and ion perturbation.

\section{Material and Methods}

The sample preparation, cleaning and grinding, of the Nafion ${ }^{\circledR} 112$ (Ion Power Inc.) has already been described elsewhere $[15,16]$. Briefly, the sample was boiled in $\mathrm{H}_{2} \mathrm{O}_{2}, 0.5 \mathrm{M} \mathrm{H} \mathrm{H}_{2} \mathrm{SO}_{4}$ and sonicated in $50 \%$ ethanol solution and rinsed thoroughly, then frozen into liquid nitrogen and ground. The dry sample was kept for several hours under vacuum, and then progressively hydrated, so that the quantity of dry Nafion was constant in all the samples. The hydration level is determined by $\lambda$, which is the number of mole of water per mole of sulfonate group. The characteristic confinement size is directly related to the hydration and vary between 2 and $6 \mathrm{~nm}$ for $\lambda$ ranging from 11 to 40 respectively, as shown in figure 1.

The technical details about the Neutron Diffraction on the diffractometer D16 at the Institut Laue Langevin (France) were also described in the reference [16]. The sample used in this experiment had a hydration level $\lambda \sim 40$.

The X-ray Compton measurements were performed on a laboratory setup at the Physics and Geology Department of the University of Perugia, in an aluminium cell with $0.25 \mu \mathrm{m}$ thickness Beryllium windows, at room temperature. The sample was $5 \mathrm{~mm}$ thick, and hydration varying between $\lambda=14$ to 35. The Compton profile was measured at a scattering angle of $\theta=123^{\circ}$ using the $\mathrm{Ag} K_{\alpha}$ radiation with an energy of $20.104 \mathrm{keV}$, and a silicon drift detector AMPTEK XR-100T/CR. The instrumental resolution at the sample 
position has a FWHM of 2.08 a.u.

The Compton profiles are determined in the impulse approximation and the non-relativistic limit, and no corrections for multiple scattering were made in this first study. The empty cell was subtracted to the bulk water sample with an average transmission of 0.5. The intensity issued from the Compton process is measured as a function of energy, and further converted in momentum transfer to obtain the projection of the Compton Profile along the scattering vector direction $z: J(q)$ from the relations $p=\frac{m}{q} \omega-\frac{q}{2}=$ $m \frac{\omega 1-\omega 2-\frac{\omega 1 \omega 2}{m}(1-\cos (\theta))}{\sqrt{\left.\omega 1^{2}+\omega 2^{2}-2 \omega 1 \omega 2 \cos (\theta)\right)}}$ with $\omega=\omega 2-\omega 1$, where $\omega 1$ and $\omega 2$ are respectively the initial and final energies of the scattered photon.

\section{Results and Discussion}

\section{Neutron diffraction measurements}

Neutron diffraction on the powder of hydrated membranes enabled the monitoring of ice the formation during the temperature cycle. Upon cooling, ice progressively forms, and melts reversibly upon heating. The positions of the three main Bragg diffraction peaks observed, (100), (002) and (101) enable the unambiguous assignment of the crystalline phase to the one of hexagonal ice (Ih). The temperature dependence of the peak positions can also be extracted from the data, and is shown in figure 2. The slope gives the thermal expansion coefficient. In a linear approximation, it is found to be $50.3,48.6$ and $47.5 \pm 2.0 \cdot 10^{-6} K^{-1}$ respectively from the slope of the (100), (002) and (101) peaks, in very good agreement with the coefficient usually found in the literature. This absence of anomaly therefore confirms that the anomalous thermodynamic features of external ice, that does not 


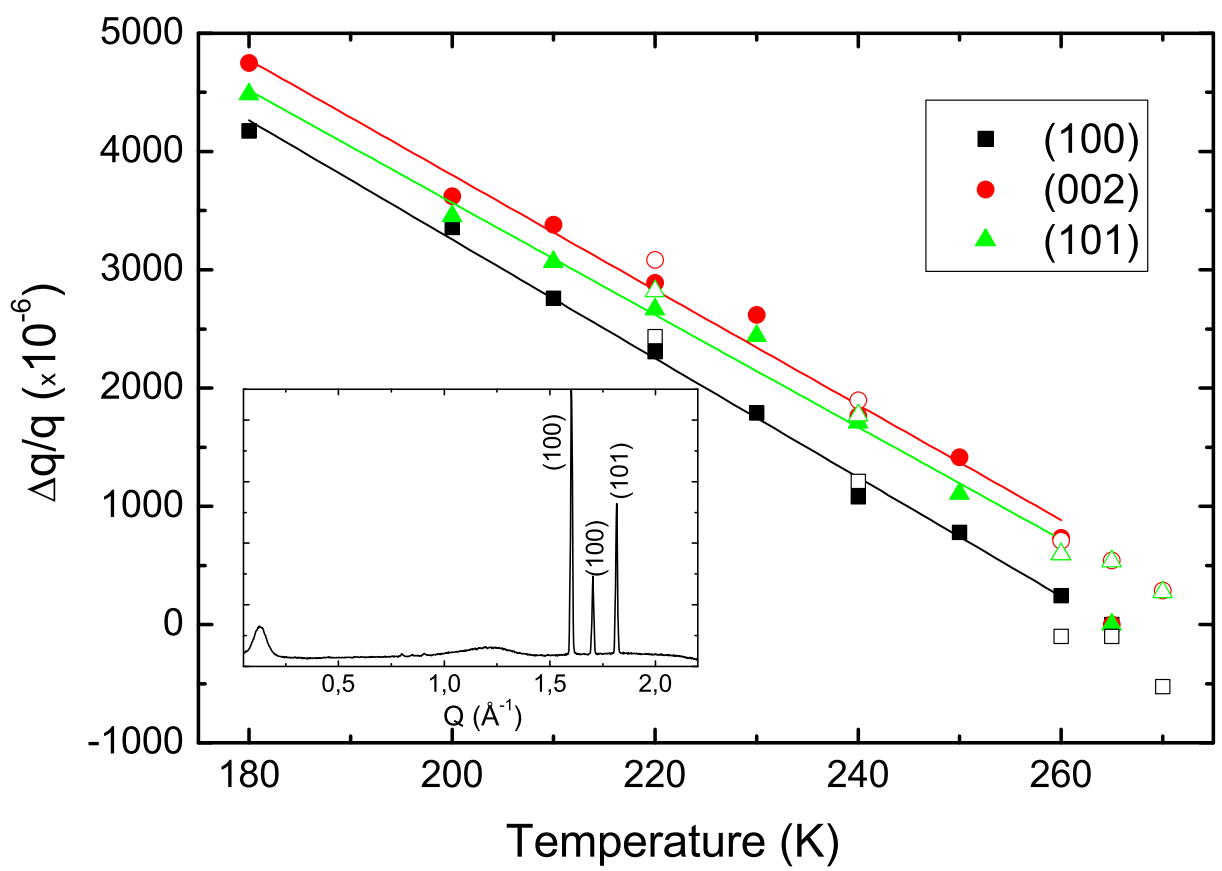

Figure 2: Temperature dependence of the relative positions of the Bragg peaks (100), (002) and (101) during cooling (plain symbols) and heating (empty symbols) of the fully hydrated Membrane, with respect to the position at $265 \mathrm{~K}$ upon cooling. The inset shows the full diffraction pattern measured at $265 \mathrm{~K}$.

show a sharp melting temperature, is due to the close contact with a complex nano-porous surface. According to our understanding, the contact with the internal liquid water induces the anomalous melting behaviour, and the amount of external ice is regulated by the chemical potential of water inside the membrane and its difference with the one of ice. Nevertheless, these experimental results on thermal expansion suggest that the crystal lattice of ice is very weakly modified by the contact with the Nafion surface and internal water. Hence, the network distortion must be limited to only few, maybe one, ice layers. 


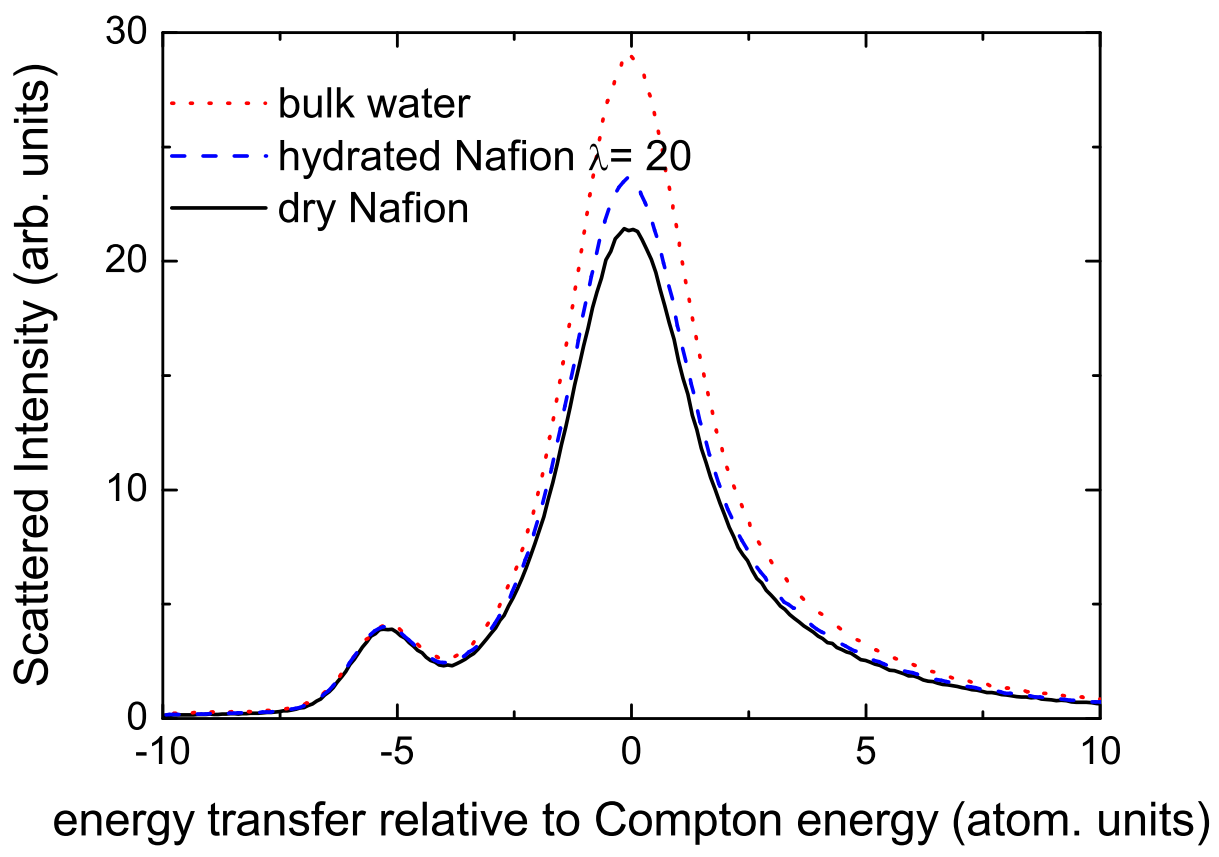

Figure 3: Inelastically scattered X-ray intensity from dry and hydrated membrane, normalised only to incident intensity. The energy transfer is calculated from the Compton energy. The elastic peak appears at -5.2 atomic units. Only the right part of the spectrum, not distorted by the presence of the elastic line, is considered for the analysis.

\section{Compton measurements}

The Compton profile $(\mathrm{CP})$, commonly named $\mathrm{J}(\mathrm{q})$, is related to the momentum distribution of the electrons of the system. It essentially provides information on the valence electron distribution, as the core electrons produce a fairly broad contribution to $\mathrm{J}(\mathrm{q})$. Therefore the $\mathrm{CP}$ is more sensitive to minor changes of the electrons which are responsible for the water molecule bonding. In particular $\mathrm{J}(0)$ is a rough measure of the wavefunction broadening and fluctuations.

The CP of hydrated membranes were measured at various hydration lev- 
els, in the liquid state at room temperature. The full spectra are presented in the figure 3 . In order to investigate the water properties, the signal of the dry membrane was subtracted to the signal of the hydrated one, while the empty cell was subtracted to the pure water sample.

The CP of the confined water at various hydration can then be compared with the CP of the pure water, as can be observed in the figure 4 .

The CP follows a progressive variation from the lowest hydration to the highest one. For the highest hydration, the profile is almost identical to the one of pure water. This corresponds to $\lambda=35$ water molecules per sulfonate group and to a characteristic confinement size of about $5 \mathrm{~nm}$. Under these conditions, our experiment can no more detect structural differences between the hydronium solution confined in Nafion and the bulk water.

At lower hydration, instead, differences in $\mathrm{CP}$ can be observed between confined and bulk water. The strongest differences are observed for the lowest hydration $\lambda=14$, in which the confinement radius is about $2 \mathrm{~nm}$. At this point, the origin of the variation in $\mathrm{CP}$ can either be assigned to the presence of ions, or distortions of the hydrogen bond network of water under confinement. Properties of the hydrogen bond network can also be accessed by Infra Red spectroscopy. Several works were reported at various hydration contents between $\lambda=1$ to $14[25,26]$. They show an evolution of the band positions when hydration increases toward the spectrum characteristic of bulk water, although even at $\lambda=14$, slight differences are still observed with respect to bulk water.

Recent Compton Scattering studies [27] were performed on membranes also hydrated at $\lambda=14$. The CP changes observed in our study are in full 
agreement with this previous work, in which the authors ruled out the effect of disorder created by the ions, on the basis of other studies of ionic solutions [23]. They estimated that the presence of ions gives a negligible contribution to the $\mathrm{CP}$ variations, and assigned their experimental observations only to confinement effects. However, they did not investigate the concentration (that is hydration level) dependence. Indeed, the concentration increases when the confinement size decreases: both these effects contribute together versus a larger distortion of the hydrogen bond network at lower hydration. Further studies, e.g. experimental investigations of water and electrolytes confined in simple matrices and the study of the temperature dependence of $\mathrm{CP}$, are therefore needed to discriminate between the two possible origins.

\section{Conclusion}

We examined the properties of the hydrogen bond network in hydrated Nafion, in ice outside the membrane at lower temperature by neutron diffraction, and in liquid water inside the membrane at room temperature by CS. Careful examination of the diffraction patterns of ice formed on the Nafion membrane surface (i.e. not confined in Nafion pores) did not exhibit any anomaly in the temperature behaviour, which emphasizes the role of the membrane in the sorption/desorption process. However, the Compton profile exhibits interesting variation upon hydration, showing that fluctuations in liquid water evolves as a function of the water content of the membrane. Further studies, in particular at low temperature, will be carried on to investigate the temperature dependence of this liquid state. 


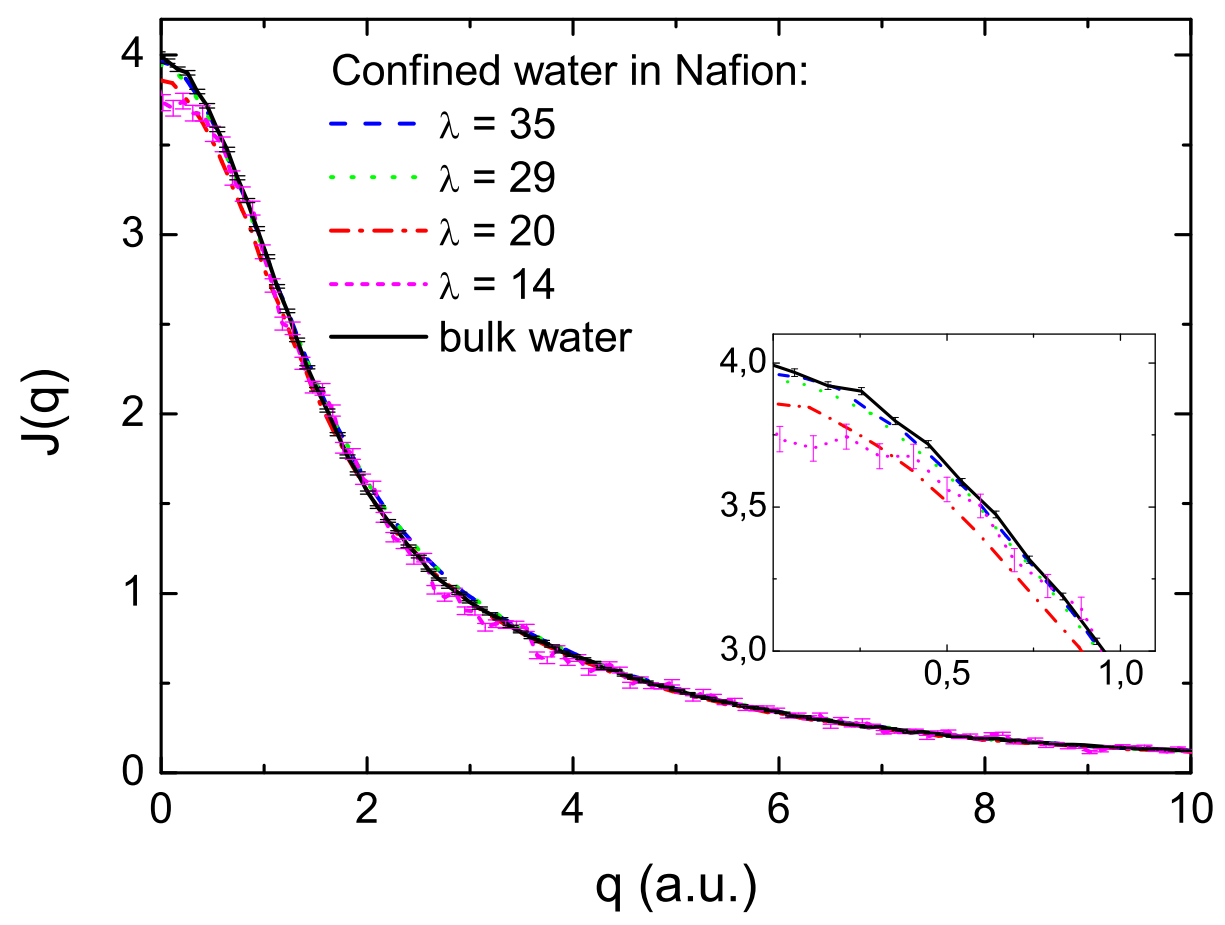

Figure 4: Compton profiles J(q) of the hydrated Nafion samples at several hydrations after subtraction of the dry sample as compared to bulk water. Only error bars for the profiles measured with best statistics (bulk water) and the poorest statistics $(\lambda=14$, due to the subtraction of the proportionally strong dry membrane signal) are represented for clarity. 


\section{Acknowledgement}

We thank the H2FC European Research Infrastructure project 2011 for funding.

\section{References}

[1] A. Thomas, Angewandte Chemie (International ed. in English) 49 (2010) $8328-44$.

[2] P. Ball, Chemical reviews 108 (2008) 74-108.

[3] H. Christenson, Journal of Physics: Condensed Matter 13 (2001).

[4] A. Taschin, P. Bartolini, A. Marcelli, R. Righini, R. Torre, Faraday Discussions 167 (2013) 293.

[5] A. Taschin, P. Bartolini, A. Marcelli, R. Righini, R. Torre, J. of Physics: Cond. Matter 27 (2015) 194107.

[6] C. Heitner-Wirguin, Journal of Membrane Science 120 (1996) 1-33.

[7] A.-C. Dupuis, Progress in Materials Science 56 (2011) 289-327.

[8] K. Mauritz, R. Moore, Chemical reviews (2004).

[9] T. D. Gierke, G. E. Munn, F. C. Wilson, Journal of Polymer Science Part B-Polymer Physics 19 (1981) 1687-1704.

[10] W. Hsu, T. Gierke, Journal of Membrane Science 13 (1983) 307-326.

[11] G. Gebel, Polymer 41 (2000) 5829-5838. 
[12] A.-L. Rollet, O. Diat, G. Gebel, The Journal of Physical Chemistry B 106 (2002) 3033-3036.

[13] K. Schmidt-Rohr, Q. Chen, Nature Materials 7 (2008) 75-83.

[14] M. Cappadonia, J. Erning, U. Stimming, Journal of Electroanalytical Chemistry 376 (1994) 189-193.

[15] M. Plazanet, P. Bartolini, R. Torre, C. Petrillo, F. Sacchetti, The Journal of Physical Chemistry. B 113 (2009) 10121-7.

[16] M. Plazanet, F. Sacchetti, C. Petrillo, B. Demé, P. Bartolini, R. Torre, Journal of Membrane Science 453 (2014) 419-424.

[17] M. Escoubes, M. Pineri, E. Robens, Thermochimica Acta 82 (1984) 149-160.

[18] M. Pineri, F. Volino, M. Escoubes, Journal of Polymer Science Part B-Polymer Physics 23 (1985) 2009-2020.

[19] M. Pineri, G. Gebel, R. Davies, O. Diat, Journal of Power Sources 172 (2007) 587-596.

[20] A. Guillermo, G. Gebel, H. Mendil-Jakani, E. Pinton, The journal of physical chemistry. B 113 (2009) 6710-7.

[21] H. Mendil-Jakani, R. J. Davies, E. Dubard, A. Guillermo, G. Gebel, Journal of Membrane Science 369 (2011) 148-154.

[22] C. Bellin, B. Barbiellini, S. Klotz, T. Buslaps, G. Rousse, T. Str, A. Shukla 094117 (2011) 1-5. 
[23] K. Nygård, M. Hakala, S. Manninen, K. Hämäläinen, M. Itou, A. Andrejczuk, Y. Sakurai, Physical Review B (2006) 1-9.

[24] T. W. Martin, Z. S. Derewenda, Nature Structural Biology 6 (1999) 403-406.

[25] D. E. Moilanen, I. R. Piletic, M. D. Fayer, The Journal of Physical Chemistry A 110 (2006) 9084-9088.

[26] S. Dalla Bernardina, J.-b. Brubach, Q. Berrod, A. Guillermo, P. Judeinstein, P. Roy, S. Lyonnard, The Journal of Physical Chemistry C 118 (2014) 25468-25479.

[27] G. F. Reiter, A. Deb, Y. Sakurai, M. Itou, V. G. Krishnan, S. J. Paddison, Physical Review Letters 036803 (2013) 1-5. 